\title{
CARACTERÍSTICAS FÍSICAS, FÍSICO-QUÍMICAS E SENSORIAIS DA ÁGUA DE FRUTOS DE COQUEIRO ANÃO VERDE ORIUNDO DE PRODUÇÃO CONVENCIONAL E ORGÂNICA ${ }^{1}$
}

\author{
Physical, physical-chemical and sensorial characteristics of coconut water from \\ green-dwarfed coconut palm from conventional and organic production
}

\author{
Daniel Lima Verde da Silva' ${ }^{2}$, Ricardo Elesbão Alves ${ }^{3}$, Raimundo Wilane de Figueiredo ${ }^{4}$, \\ Vlayrton Tomé Maciel², Josefranci Moraes de Fariass, Antônio Renes Lins de Aquino ${ }^{3}$
}

\begin{abstract}
RESUMO
Neste trabalho, objetivou-se comparar a qualidade da água de coco obtida de frutos de coqueiro anã verde, produzidos em dois sistemas distintos de produção: convencional e orgânico, mediante as análises físicas (cor da casca, peso, volume, comprimento e diâmetro), físico-químicas (sólidos totais, acidez titulável, $\mathrm{pH}$, turbidez, açúcares solúveis totais e redutores) e os minerais: (Ca, $\mathrm{Mg}$, $\mathrm{Na}, \mathrm{K}, \mathrm{Fe}, \mathrm{Mn}, \mathrm{P}$, sulfato, cloreto e C.E.); e análise sensorial. Foram realizados os seguintes parâmetros sensoriais: avaliação global, turbidez, doçura e sabor. Os frutos utilizados nesse experimento encontravam-se com 210 dias após a polinização das inflorescências e foram provenientes de dois plantios comerciais do município de Trairí- Ceará. O experimento foi desenvolvido no laboratório de Fisiologia e Tecnologia de Pós-Colheita da Embrapa Agroindústria Tropical, Fortaleza-CE. Foi observado, a partir dos resultados das análises físicas, que o coco produzido em sistema convencional é melhor do que os produzidos organicamente. Para todas as variáveis estudadas, porém apenas as medições de comprimento e peso mostraram diferenças importantes por meio do teste Tukey em um nível de 5\% de probabilidade. Nas análises físico-químicas e análise sensorial não houve diferença significativa para os tratamentos.
\end{abstract}

Termos para indexação: Cocus nucifera L., água de coco, pós-colheita, produção convencional e orgânica.

\section{ABSTRACT}

This work aimed to assess and compare the quality of coconut water produced in two different systems: standard and organic. The physical characteristics of the fruit were described (skin color, weight, water volume, length, and diameter), the physicalchemical characteristics of the water were assessed (total solid content, total titrable acidity, $\mathrm{pH}$, turbidity, reducing and total soluble sugars), the mineral content was checked (Fe, $\mathrm{P}, \mathrm{K}, \mathrm{Na}, \mathrm{Ca}, \mathrm{Mg}, \mathrm{Mn}$, sulphate, chloride, and C.E.), and the sensory analysis was conducted assessing the following parameters: overall evaluation, turbidity, sweetness, and flavor. The fruits used in this experiment were harvested 210 days after the pollination of the flowers and were from two commercial plantations at the municipality of TrairíCeara.The experience was developed at the Physiology and Technology Post-Harvest Laboratory of Embrapa Tropical Agroindustry, Fortaleza-CE. We observed, from the results of the physical analysis, that the coconut produced in the standard system is better than that produced organically, for all of the studied variables. However, only the measurements of length and weight showed important differences through the Tukey test at the level of 5\% probability. The physical-chemical and sensory analysis did not show significant differences between the treatments.

Index terms: Cocus nucifera L., coconut water, postharvest, conventional and organic production.

(Recebido em 27 de outubro de 2006 e aprovado em 9 de abril de 2009)

\section{INTRODUÇÃO}

As frutas constituem fonte nutricional de vitaminas, minerais e carboidratos solúveis. No entanto, algumas apresentam teor mais elevado de um ou outro nutriente (Matsuura \& Rolim, 2002).

A preocupação com a saúde e a forma física tem-se apoiado também nas bebidas naturais, que visam a repor as perdas de água, vitaminas e sais minerais sofridas durante grande esforço físico no trabalho, em esporte e divertimentos. Desta forma, existe uma atração dos consumidores por novos produtos e uma tendência por sabores exóticos, naturais, ligados à saúde, a exemplo da água de coco (Cuenca et al., 2002).

A água de coco é uma bebida natural, pouco calórica, com sabor agradável, conhecida mundialmente e muito apreciada em todo o Brasil, principalmente nas regiões litorâneas (Penha, 1998; Aragão, 2000; Aroucha \&

\footnotetext{
${ }^{1}$ Apoio financeiro CNPq

Engenheiro Agrônomo pela Universidade Federal do Ceará. CP 12168, 60356-000, Fortaleza, CE.

${ }^{3}$ Pesquisador da Embrapa Agroindústria Tropical, CP 3761, 60511-110, Fortaleza, CE, elesbao@pesquisador. cnpq.br

4Professor do Departamento de Tecnologia de Alimentos, Universidade Federal do Ceará, figueira@ufc.br.

${ }^{5}$ Aluna do curso de mestrado em Ciência e Tecnologia de Alimentos da Universidade Federal do Ceará. Fortaleza, CE. Bolsista FUNCAP, josefranci_farias@yahoo.com.br
} 
Vianni, 2002), sendo usada há séculos, para saciar a sede, como substituto da água, e também para repor eletrólitos nos casos de desidratação (Aragão et al., 2001). Os eletrólitos podem ser sais inorgânicos simples de sódio, potássio ou magnésio ou moléculas orgânicas complexas (Whitmire, 2002).

Por ser boa fonte de potássio, cloreto e cálcio, a água de coco pode ser a melhor indicação em situações específicas de carência em que o teor desses eletrólitos necessita ser aumentado (Matsui, 2006).

A fruticultura orgânica ainda encontra-se bastante incipiente, o que resulta em oferta irregular de produtos nas prateleiras dos supermercados e nas feiras orgânicas. No entanto, o crescimento do mercado brasileiro para o consumo de produtos orgânicos tem sido significativos, com taxa média anula de $22,5 \%$. Na agricultura orgânica, as frutas ocupam a maior área plantada, correspondente a $11 \%$ do total (30 mil hectares) e 3,9\% dos produtores (Borges et al., 2003).

Embora existam hoje inúmeros produtos de origem orgânica, verifica-se um maior crescimento nas áreas de produção para consumo in natura, na horticultura e na fruticultura. Nesse sentido, a cultura do coqueiro, recentemente vem se destacando também nesse tipo de produção. Atualmente, existem diversas áreas cultivadas com coqueirais orgânicos, gerando um grande interesse por parte de muitos produtores, inclusive daqueles que já produzem coco de forma convencional (Lopes, 2005).

De acordo com Mazenotti (2007), o maior problema enfrentado pelas empresas que exportam água de coco para a Europa é a conservação do produto.

Neste trabalho, objetivou-se comparar por meio de análises físicas, físico-químicas, químicas e sensoriais, a qualidade da água de frutos de coqueiro anão verde produzido nos sistemas convencional e orgânico.

\section{MATERIAL E MÉTODOS}

Frutos da variedade anã verde foram colhidos em plantios comerciais, com sistemas de produção distintos (orgânico e convencional), sendo ambos localizados no município de Trairí-Ce. Os pomares foram formados há sete anos, sendo os frutos colhidos com aproximadamente sete meses de idade. Foram selecionadas 12 plantas (repetições) aleatoriamente de cada sistema (tratamento) e coletados cinco frutos/planta, ou seja, 60 frutos de cada tratamento, totalizando 120 frutos.

Após a colheita, os frutos foram transportados para o Laboratório de Fisiologia e Tecnologia de Pós-colheita da Embrapa Agroindústria Tropical em Fortaleza, CE, onde foram realizadas as avaliações físicas dos frutos, peso, obtido por pesagem individual, no dia da colheita, utilizando-se balança semianalítica de marca Mark com capacidade para $3100 \mathrm{~g}$, com os resultados expressos em gramas (g); comprimento e diâmetro, realizados com auxílio de paquímetro digital, modelo Sylvac com escala graduada em milímetro; volume de água determinado, individualmente em cada fruto, utilizando-se proveta graduada de $1000 \mathrm{~mL}$; e cor da casca (L, a, b), realizada por meio da média de duas leituras efetuadas em pontos aproximadamente equidistantes, utilizando-se colorímetro da marca Minolta modelo CR 300. As avaliações físicoquímicas na água constaram de: sólidos solúveis totais (SST), por meio da leitura em refratômetro digital de bancada modelo Atago PR 101, escala 0 a $45^{\circ} \mathrm{C}$, com compensação automática de temperatura, sendo os resultados expressos em \% (AOAC, 1992); pH, determinado a partir da própria água com potenciômetro equipado com eletrodo de membrana de vidro. Em seguida, as amostras foram diluídas na proporção de 1:50 e a acidez total titulável (\% de ácido málico) foi determinada, utilizando-se titulador automático, com solução de $\mathrm{NaOH} 0,1 \mathrm{~N}$, conforme Instituto Adolfo Lutz (1985); relação SST/ATT, obtida por meio do quociente entre os duas variáveis; turbidez, determinada por meio do turbidímetro, sendo os resultados expressos em unidade nefelométrica (n.t.u.); para os açúcares solúveis totais (AST), utilizou-se o método de Antrona de acordo com Yemn \& Willis (1954) e para os açúcares redutores (AR), utilizou-se solução de ácido dinitrosalicílico (DNS), conforme descrito por Miller (1959); condutividade elétrica procedeu-se leitura em condutivímetro e os resultados foram expressos em $\mathrm{ms} / \mathrm{cm}$.

Para a determinação dos minerais, seguiram-se as metodologias indicadas por Silva (1999). As amostras de água de coco foram submetidas à digestão úmida em solução composta por ácido nítrico e ácido perclórico na proporção $\mathrm{HNO}_{3}: \mathrm{HClO}_{4}(3: 1 \mathrm{v} / \mathrm{v})$, conforme descrito por Silva (1999). Ferro (Fe), Cálcio (Ca), Magnésio (Mg), Manganês $(\mathrm{Mn})$, foram determinados por meio de espectrofotometria de absorção atômica, em equipamento de marca Perkin-Elmer (modelo A-Analyst) 300, com chama ar/acetileno nas proporções recomendadas pelo fabricante do instrumento para os diferentes elementos. Os comprimentos de onda utilizados para esses minerais estão de acordo com Silva (1999). Os minerais Potássio (K) e Sódio (Na) foram analisados por fotometria de chama, em Fotômetro de Chama marca Digimed, modelo DM-61. Para o Fósforo (P) e Sulfatos procederam-se leituras em espectrofotômetro de marca Femto modelo 600 plus e os resultados expressos em $\mathrm{mg} / \mathrm{L}$. Construiu-se uma curva padrão para cada mineral, conforme Silva (1999). Para 
determinação de Cloreto, foram transferidos $5 \mathrm{~mL}$ de amostra para um frasco de erlenmeyer de $125 \mathrm{~mL}$, adicionando-se em seguida 3 gotas de cromato de potássio $\left(\mathrm{K}_{2} \mathrm{CrO}_{4} 5 \%\right)$ agitando-o, e titulou-se com solução de nitrato de prata $\left(\mathrm{AgNO}_{3} 0,05 \mathrm{~N}\right)$ até a formação de um precipitado de coloração avermelhada, observando-se o volume gasto.

A avaliação sensorial das amostras de água de coco foi realizada no Laboratório de Análise Sensorial da Embrapa Agroindústria Tropical, em Fortaleza-CE, aplicados a sessenta provadores não treinados. Empregouse o teste de aceitação mediante os atributos de avaliação global, turbidez, doçura e sabor, com utilização de escala hedônica estruturada de nove pontos, em que o nove representava a nota máxima "gostei muitíssimo", e um a nota mínima "desgostei muitíssimo" (Stone \& Sidel, 1993). Os frutos foram abertos para a retirada da água, a qual foi homogeneizada à temperatura ambiente $\left(28^{\circ} \mathrm{C}\right)$, posteriormente resfriada até $12^{\circ} \mathrm{C}$, codificada em números de três dígitos e colocados em copos descartáveis de 50 $\mathrm{mL}$ e servidos a 60 provadores não treinados. Cada provador recebeu as duas amostras, servidas em bandejas plásticas, juntamente com um copo de água, para que este fosse utilizado pelo provador entre uma e outra degustação das amostras, segundo a metodologia proposta por Meilgaard et al. (1987). As análises foram realizadas em cabines individuais.

Para efeito de análise estatística das avaliações físico-químicas e químicas utilizou-se um delineamento inteiramente ao acaso com três repetições. Já para análise sensorial o delineamento foi o de blocos ao acaso, sendo cada provador considerado um lote. Os resultados físicos do fruto e físico-químicos, químicos e sensoriais da água de coco foram tratados estatisticamente mediante a análise de variância (ANOVA) e aplicado o teste de Tukey entre as médias a $5 \%$ de probabilidade, por meio do programa estatístico SAS Institute (1996).

\section{RESULTADOS E DISCUSSÃO}

Pela falta de informações disponíveis na literatura no que diz respeito à água de coco oriunda de produção orgânica, conduziu-se este trabalho, com base em fontes desses produtos cultivados em sistema de produção convencional.

Os valores encontrados na Tabela 1 mostram que, entre as características físicas analisadas, apenas comprimento e peso médio dos frutos, diferiram significativamente $(\mathrm{p}<0,05)$ entre os sistemas de cultivos.

Para o peso dos frutos, coco produzido convencional $(2209,91 \mathrm{~g})$ foi superior ao orgânico $(1984,93)$. Aragão et al. (2002) e Camboim Neto (2002) em estudos
Tabela 1 - Caracterização física de frutos de coqueiro anão verde com sete meses de idade, oriundos de diferentes sistemas de cultivos.

\begin{tabular}{lcc}
\hline \multirow{2}{*}{ Análises } & \multicolumn{2}{c}{ Sistemas de Produção } \\
\cline { 2 - 3 } & Orgânico & Convencional \\
\hline Peso $(\mathrm{g})$ & $1984,93 \mathrm{a}$ & $2209,91 \mathrm{~b}$ \\
\hline Comprimento $(\mathrm{mm})$ & $165,77 \mathrm{a}$ & $172,32 \mathrm{~b}$ \\
\hline Diâmetro $(\mathrm{mm})$ & $148,99 \mathrm{a}$ & $149,72 \mathrm{a}$ \\
\hline Volume de água $(\mathrm{mL})$ & $425,16 \mathrm{a}$ & $467,16 \mathrm{a}$ \\
\hline $\begin{array}{l}\text { Relação Água/Fruto } \\
\text { g/mL) }\end{array}$ & $0,21 \mathrm{a}$ & $0,21 \mathrm{a}$ \\
\hline Cor da Casca $\left(\mathrm{a}^{*}\right)$ & $-14,72 \mathrm{a}$ & $-14,48 \mathrm{a}$ \\
\hline Cor da Casca $\left(\mathrm{b}^{*}\right)$ & $30,42 \mathrm{a}$ & $30,28 \mathrm{a}$ \\
\hline Cor da Casca $\left(\mathrm{L}^{*}\right)$ & $52,50 \mathrm{a}$ & $51,86 \mathrm{a}$ \\
\hline
\end{tabular}

* Médias seguidas da mesma letra na mesma linha não diferem entre si, pelo teste de Tukey, ao nível de 5\% de probabilidade.

com coqueiros da variedade anã verde, com sete meses de idade, encontraram valores um pouco menores, com uma média de 1759 g e 1623,7 g, respectivamente. Laguna (1996) encontrou valores (1937 g) semelhantes ao presente trabalho. Já, Araújo (2003), nas mesmas condições citadas, encontrou valores médios de aproximadamente $2450 \mathrm{~g}$.

Os valores obtidos para comprimento do fruto estão próximos aos de outros pesquisadores. Em cocos da variedade anã verde com sete meses de idade encontramse Camboim Neto (2002) (166 mm), Maciel (2008) (165mm) e Emparn (2001) (186 mm), porém abaixo daqueles encontrados por Araújo (2003) (205mm). Não houve diferença significativa quanto ao diâmetro dos frutos pelo teste de Tukey ao nível de 5\% de probabilidade. Esses valores estão abaixo do constatado por Araújo (2003), em coqueiros da variedade anã verde com sete meses que encontrou diâmetros médios de 166 mm, e bem acima dos encontrados por Empresa de Pesquisa Agropecuária do Rio Grande do Norte (2001) e Camboim Neto (2002) que foram de 139,4 mm e $139 \mathrm{~mm}$, respectivamente.

Os volumes de água de coco encontrados em frutos oriundos de cultivos orgânico e convencional, 425,16 e $467,16 \mathrm{~mL}$, respectivamente (Tabela 1), estão bem acima do limite mínimo recomendado por Assis et al. (2000), para colheita de frutos de coqueiro anão verde com sete meses de idade, cujos valores podem variar entre 350 e $450 \mathrm{~mL}$ de água. Ao analisar frutos de coqueiro anão em seis cultivares selecionadas, durante seu período de desenvolvimento, Tavares et al. (1998) e Maciel (2008) encontraram resultados bem menores de volume de água, de 101 a $310 \mathrm{~mL}$, respectivamente. Segundo Jackson et al. (2004), a 
quantidade de água dos frutos de coqueiro sofre influência da cultivar e do estádio de maturação.

Na Tabela 2, os valores encontrados de sólidos solúveis totais, $\mathrm{pH}$, acidez total titulável, relação SST/ATT, turbidez e açúcares não diferiram significativamente entre os sistemas de produção, apresentaram-se semelhantes aos resultados obtidos por Tavares et al. (1998), Aragão et al. (2001) e Camboim Neto (2002), em frutos da variedade anã verde com sete meses de idade.

Tabela 2 - Caracterização físico-química e química da água de coco de frutos da variedade anã verde com sete meses de idade, oriunda de diferentes sistemas de cultivo.

\begin{tabular}{lcc}
\hline \multirow{2}{*}{ Análises } & \multicolumn{2}{c}{ Sistemas de Produção } \\
\cline { 2 - 3 } & Orgânico & Convencional \\
\hline SST ( ${ }^{\circ}$ Brix) & $6,0 \mathrm{a}$ & $5,58 \mathrm{a}$ \\
\hline pH & $4,94 \mathrm{a}$ & $4,96 \mathrm{a}$ \\
\hline Acidez (\% ácido & & $0,064 \mathrm{a}$ \\
málico) & $0,058 \mathrm{a}$ & \\
\hline Turbidez (n.t.u) & $13,80 \mathrm{a}$ & $12,08 \mathrm{a}$ \\
\hline Açúcares Solúveis & & $4,66 \mathrm{a}$ \\
Totais $(\%)$ & $4,71 \mathrm{a}$ & \\
\hline Açúcares Redutores $(\%)$ & $4,02 \mathrm{a}$ & $4,29 \mathrm{a}$ \\
\hline Relação SST/ATT & $105,30 \mathrm{a}$ & $93,17 \mathrm{a}$ \\
\hline C.E. $(\mathrm{ms} / \mathrm{cm})$ & $5,67 \mathrm{a}$ & $4,82 \mathrm{~b}$ \\
\hline Na (mg/100 mL) & $9,17 \mathrm{a}$ & $7,67 \mathrm{a}$ \\
\hline K (mg/100 mL) & $173,50 \mathrm{a}$ & $152,00 \mathrm{a}$ \\
\hline Fe (mg/100 mL) & $0,15 \mathrm{a}$ & $0,14 \mathrm{a}$ \\
\hline Mn (mg/100 mL) & $0,44 \mathrm{a}$ & $0,19 \mathrm{~b}$ \\
\hline Ca (mg/100 g) & $47,98 \mathrm{a}$ & $31,95 \mathrm{~b}$ \\
\hline Mg (mg/100 mL) & $15,01 \mathrm{a}$ & $13,93 \mathrm{a}$ \\
\hline P (mg/100 g) & $3,92 \mathrm{a}$ & $3,31 \mathrm{a}$ \\
\hline Cloretos & $149,46 \mathrm{a}$ & $163,56 \mathrm{a}$ \\
\hline Sulfatos & $5,67 \mathrm{a}$ & $4,82 \mathrm{a}$ \\
\hline
\end{tabular}

* Médias seguidas da mesma letra na mesma linha não diferem entre si, pelo teste de Tukey, ao nível de 5\% de probabilidade.

Dentre os minerais analisados (Tabela 2), apenas cálcio e manganês diferiram significativamente. Os teores de minerais obtidos estão coerentes aos encontrados por Tavares et al. (1998) e Rosa \& Abreu (2000) com exceção do $\mathrm{Ca}, \mathrm{Mg}$ e Fe, que obtiveram valores bem superiores. As diferenças encontradas entre os tratamentos quanto aos minerais, podem ser explicadas pelas diferenças dos metabolismos fisiológico e bioquímico, o que faz com que respondam de maneira diferente às condições edafoclimáticas da região (Araújo, 2003).

Segundo Brasil (2003), os requisitos utilizados para caracterizar a qualidade da água de coco quanto às características sensoriais são: apresentar cor característica, sabor levemente adocicado, aroma próprio e aparência variando do translúcido para o opaco, sendo que a presença de pequena quantidade de partículas sobrenadantes da polpa do coco não desqualifica o produto.

Segundo Maciel (2008), os alimentos, quer sejam industrializados ou não, estão em constante atividade biológica, o que se manifesta por alterações na natureza química, física e na atividade microbiológica ou enzimática. Tais alterações prejudicam sua qualidade, principalmente no caso dos alimentos de origem vegetal que, mesmo quando retirados das plantas, continuam respirando e assim realizando o metabolismo. Isso pode se caracterizar pela infestação por microorganismos, insetos ou pela presença de certos contaminantes químicos, os quais causam a perda de certos atributos específicos, tais como a cor, sabor, textura e viscosidade.

A aceitabilidade da água de coco está diretamente relacionada com os atributos de qualidade, tais como a doçura, a turbidez e, consequentemente, com o sabor agradável (Minim, 2006). Não houve diferença significativa quanto à análise sensorial. A água de coco produzida em dois sistemas distintos de cultivo apresentou características desejáveis para o consumo in natura, vindo as notas do cultivo orgânico apresentando-se ligeiramente superiores ao convencional (Tabela 3).

Segundo Chitarra \& Chitarra (2005) a doçura dos frutos é resultante do balanceamento das concentrações de diversos açúcares e dos ácidos orgânicos presentes no caso específico da água de coco, o ácido málico. De acordo com Kays (1991) o sabor está diretamente ligado às mudanças na acidez.

Tabela 3 - Análise sensorial da água de coco da variedade anã verde com sete meses de idade.

\begin{tabular}{ccc}
\hline \multirow{2}{*}{ Análises } & \multicolumn{2}{c}{ Sistemas de Produção } \\
\cline { 2 - 3 } & Orgânico & Convencional \\
\hline Aceitação global & $7,52 \mathrm{a}$ & $7,31 \mathrm{a}$ \\
\hline Turbidez & $3,27 \mathrm{a}$ & $2,93 \mathrm{a}$ \\
\hline Doçura & $4,20 \mathrm{a}$ & $3,93 \mathrm{a}$ \\
\hline Sabor & $4,90 \mathrm{a}$ & $4,40 \mathrm{a}$
\end{tabular}

*Médias seguidas da mesma letra na mesma linha não diferem entre si, pelo teste de Tukey, ao nível de 5\% de probabilidade.

\section{CONCLUSÕES}

Com exceção do peso (em torno de $10 \%$ maior para frutos produzidos em sistema convencional) e do 
comprimento, não foram observadas diferenças $(\mathrm{P}<0,05)$ para as características físicas avaliadas.

Não foram observadas diferenças $(\mathrm{P}<0,05)$ para a água dos cocos produzidos em sistemas convencional ou orgânico, levando-se em consideração as avaliações de qualidade (SST, açúcares, ATT, SST/ATT e pH) e as análises sensoriais realizadas.

De um modo geral, a água de coco produzida em sistema orgânico apresentou uma maior condutividade elétrica e, consequentemente, teores de minerais superiores.

\section{REFERÊNCIAS BIBLIOGRÁFICAS}

ARAGÃO, W.M. A importância do coqueiro anão verde. Aracaju: Embrapa Tabuleiros Costeiros, 2000. Disponível em: 〈http://www.cpatca.embrapa.br/s. Acesso em: 19 out. 2003.

ARAGÃO, W.M.; ISBERNER, I.V.; CRUZ, E.M. Água de coco. Aracajú: Embrapa Tabuleiros Costeiros, 2001. 32p (Embrapa Tabuleiros Costeiros.

Documentos, 24).

ARAGÃO, W.M.; RIBEIRO, F.E.; TUPINAMBÁ, E.A.; SIQUEIRA, E.R. de. Variedades e híbridos do coqueiro. In: ARAGÃO, W.M. (Ed.). Coco pós-colheita. Brasília, DF: Embrapa-SPI; Aracaju: Embrapa-CNPAT, 2002. p.2634. (Frutas do Brasil, 29).

ARAÚJO, M.V. Ponto de colheita e armazenamento refrigerado de coco anão verde (Cocus nucifera $L_{\text {.) }}$, sob atmosfera modificada. 2003. 61f. Dissertação (Mestrado em Fitotecnia)-Escola Superior de Agricultura de Mossoró, Mossoró, 2003.

AROUCHA, E.M.M.; VIANNI, R. Determinação de ácido ascórbico na água de coco por cromatografia líquida e pelo método titulométrico. Revista Ceres, Viçosa, v.49, n.283, p.245-251, 2002.

ASSIS, J.S. de; RESENDE, J.M.; SILVA, F.O.; SANTOS, C.R. dos; NUNES, F. Técnicas para colheita e pós-colheita do coco verde. Petrolina: Embrapa SemiÁrido, 2000. 6p. (Embrapa Semi-Árido. Comunicado técnico, 95).

ASSOCIATION OF OFFICIAL ANALYTICAL CHEMISTRY. Official methods of analysis of the association of official chemistry. Washington, DC, 1992. $1115 \mathrm{p}$.
BORGES, A.L.; TRINDADE, A.V.; SOUZA, L. da S.; SILVA, M.N.B. da. Cultivo orgânico de fruteiras tropicais: manejo do solo e da cultura. Cruz das Almas: Embrapa Mandioca e Fruticultura, 2003. 12p. (Embrapa CNPMF. Circular técnica, 64).

BRASIL. Ministério da Agricultura. Instrução normativa n' 39, de 29 de Maio de 2002. Aprova o Regulamento Técnico para fixação de identidade e qualidade da água de coco. Disponível em: 〈http//www.agricultura.gov.brh idas/ddiv/pdf/in $392002 . \mathrm{pdf}$. A Acesso em: 2 dez. 2003 .

CAMBOIM NETO, L.F. Coqueiro anão verde: influência de diferentes lâminas de irrigação e de porcentagens de área molhada no desenvolvimento, na produção e nos parâmetros físico-químicos do fruto. 2002. 112f. Tese (Doutorado em Engenharia Agrícola)-Universidade Federal de Viçosa, Viçosa, MG, 2002.

CHITARRA, M.I.F.; CHITARRA, A.B. Pós-colheita de frutos e hortaliças: fisiologia e manuseio. Lavras: ESAL/ FAEP, 2005. 782p.

CUENCA, M.A.G.; RESENDE, J.M.; SAGGIN JÚNIOR, O.J.; REIS, C.S. Mercado brasileiro do coco: situação atual e perspectivas. In: ARAGÃO, W.M. Coco: póscolheita. Brasília, DF: Embrapa Informação Tecnológica, 2002. p.18.

\section{EMPRESA DE PESQUISA AGROPECUÁRIA DO RIO}

GRANDE DO NORTE S/A. Caracterização física do fruto e composição química da água de coco de cultivares anão verde de Jequi, anão amarelo e híbrido PB 121 aos 5,6,77,8 e 9 meses de idade. In: SEMANA INTERNACIONAL DA FRUTICULTURA, FLORICULTURA E AGROINDÚSTRIA, 8., 2001, Fortaleza, CE. Anais... Fortaleza: Frutal, 2001. 102p.

INSTITUTO ADOLFO LUTZ. Normas analíticas do Instituto Adolfo Lutz: métodos químicos e físicos para análise de alimentos. 3.ed. São Paulo, 1985. v.1, 533p.

JACKSON, J.C.; GORDON, A.; WIZZARD, G.; McCOOK, K.; ROLLE, R. Changes in chemical composition of coconut (Cocos nucifera L.) water during maturation of the fruit. Journal of the Science of Food and Agriculture, Easton, v.84, p.1049-1052, 2004.

KAYS, S.J. Postharvest physiology of perishable plant products. New York: AVI, 1991. 532p. 
LAGUNA, L.E. Determinação físico-química da água de coco verde em duas variedades (Cocus nucifera $\mathrm{L}$.) coco de praia e anão. 1996. 56f. Monografia (Especialização em Produção e Reprodução de Pequenos Ruminantes)Universidade Estadual do Ceará, Fortaleza, 2002.

LOPES, J.D.S. Cultivo orgânico de coco. Disponível em: 〈http://tecnologiaetreinamento.com.br/ out. 2005.

MACIEL, V.T. Caracterização física, físico-química e enzimática de frutos de seis cultivares de coqueiro anão em diferentes estádios de desenvolvimento. 2008. 103f. Dissertação (Mestrado em Fitotecnia)-Universidade Federal do Ceará, Fortaleza, 2008.

MATSUI, K.N. Inativação de enzimas presentes na água de coco (Cocos nucifera $L$.) por processo térmico através de microondas. 2006. Tese (Doutorado)-Escola Politécnica da Universidade de São Paulo, São Paulo, 2006.

MATSUURA, F.C.A.U.; ROLIM, R.B. Avaliação da adição de suco de acerola em suco de abacaxi visando à produção de um "blend" com alto teor de vitamina C. Revista Brasileira de Fruticultura, Cruz das Almas, v.24, n.1, p.138-141, 2002.

MAZENOTTI, P. Água de coco do Brasil começa a ganhar o mundo. Brasília, DF: Agência Brasil, 2007. Disponível em: <http://www.radiobras.gov.br/入̀ Acesso em: 18 mar. 2007.

MEILGAARD, M.; CIVILLE, G.V.; CARR, B.T. Sensory evaluation tecnhniques. Flórida: CRC, 1987.

MILLER, G.L. Use of dinitrosalicylit acid reagent for determination of reducing sugars. Analytical Chemistry, Washington, v.31, n.3, p.426-428, 1959.

MINIM, V.P.R. Análise Sensorial estudos com consumidores. Viçosa, MG: UFV, 2006. 225p.
PENHA, E.M. Características do coco verde para industrialização da água e da polpa gelatinosa. In: CONGRESSO BRASILEIRO DE CIÊNCIA E TECNOLOGIA DE ALIMENTOS, 16., 1998, Rio de Janeiro, RJ. Anais... Campinas: SBCTA, 1998. CDROM.

ROSA, M.F.; ABREU, F.A.P. Água de coco: métodos de conservação. Fortaleza: Embrapa-CNPAT/Sebrae/CE, 2000. 40p. (Documentos, 37).

SILVA, F.C. Manual de análises químicas de solos, plantas e fertilizantes. Brasília, DF: Embrapa Comunicação para Transferência de Tecnologia; Rio de Janeiro: Embrapa Solos; Campinas: Embrapa Informática Agropecuária, 1999. 370p.

\section{STATISTICAL ANALISYS SYSTEM INSTITUTE. SAS} Institute Inc. Cary, 1996.

STONE, H.; SIDEL, J.L. Sensory evaluation practices. 2.ed. Florida: Academic, 1993. 338p.

TAVARES, M.; CAMPOS, N.C.; NAGATO, L.C.A.F.; LAMARDO, L.C.A.; NOMATE, E.L.; CARVALHO, M.F.H.; ARAGÃO, W.M. Estudo da composição química da água de coco-anão verde em diferentes estágios de maturação. In: CONGRESSO BRASILEIRO DE CIÊNCIA E TECNOLOGIA DE ALIMENTOS, 16., 1998, Rio de Janeiro, MG. Anais... Rio de Janeiro: SBCTAA, 1998. CDROM.

YEMN, E.W.; WILLIS, A.J. The estimation off carbohydrate in plant extracts by antrone. Biochemical Journal, Cambridge, v.57, n.2, p.504-514, 1954.

WHITMIRE, S.J. Água, eletrólitos e equilíbrio ácidobase. In: MAHAN, L.K.; SCOTT-STUMP, S.K. Alimentos, nutrição e dietoterapia. 10.ed. São Paulo: Roca, 2002. p.146-156. 